\title{
Giant thermopower and figure of merit in single-molecule devices
}

\author{
C. M. Finch, * V. M. García-Suárez, and C. J. Lambert \\ Department of Physics, Lancaster University, Lancaster LA1 4YB, United Kingdom
}

(Received 16 November 2008; published 12 January 2009)

\begin{abstract}
We present a study of the thermopower $S$ and the dimensionless figure of merit $Z T$ in molecules sandwiched between gold electrodes. We show that for molecules with side groups, the shape of the transmission coefficient can be dramatically modified by Fano resonances near the Fermi energy, which can be tuned to produce huge increases in $S$ and ZT. This shows that molecules exhibiting Fano resonances have a high efficiency of thermoelectric cooling which is not present for conventional ungated molecules with only delocalized states along their backbone.
\end{abstract}

DOI: 10.1103/PhysRevB.79.033405

PACS number(s): 85.65.+h, 72.15.Jf, 73.63.-b

Recent advances in single-molecule electronics have been underpinned by considerable improvements in electrical contacting techniques, ${ }^{1-3}$ which have allowed the identification of a range of fundamental properties including switching, ${ }^{4,5}$ rectification, ${ }^{6,7}$ memory, ${ }^{8}$ and sensing. ${ }^{9}$ Single-molecule devices offer a potential route to sub-10-nm electronics. For the purpose of designing large-scale integration of such devices, knowledge of thermal as well as electrical properties is needed. Furthermore, knowledge of thermoelectric properties of single molecules ${ }^{10}$ may underpin novel thermal devices such as molecular-scale Peltier coolers and provide new insight into mechanisms for molecular-scale transport. For example, from the sign of the thermopower it is possible to deduce the conduction mechanism or relative position of the Fermi energy, ${ }^{11}$ with a positive sign indicating $p$-type conduction, which means the Fermi energy is lying closer to the highest occupied molecular orbital (HOMO) level. The thermopower can also exhibit very interesting behaviors, such as a linear increase with the molecular length. ${ }^{12}$ Other theoretical studies have shown the possibility of changing both the sign and magnitude of the thermopower by electrically gating a molecular wire, ${ }^{13,14}$ which moves the Fermi energy across a transmission resonance and thus changes its sign.

However when the Fermi energy lies within the HOMOlowest unoccupied molecular orbital (LUMO) gap, thermopower is expected to be low. Indeed recently, Reddy et $a l .{ }^{15}$ measured the room-temperature thermopower of 1,4-biphenyldithiol (BPDT), using a modified scanning tunnel microscope. They found the thermopower to be $+12.9 \mu \mathrm{V} \mathrm{K}^{-1}$. This low value was attributed to the Fermi energy sitting within the molecule's HOMO-LUMO gap where both the density of states (DOS) and transmission coefficients are relatively flat.

In this Brief Report we show that much higher values can be obtained from molecules exhibiting Fano resonances. We investigate the thermoelectric properties of two different molecules, the BPDT and CSW-470-bipyridine (CSW-479), ${ }^{16}$ as a function of the rotation angles of some of the molecular groups. Both exhibit Breit-Wigner-type resonances in the transmission coefficient $\mathcal{T}(E)$ near the Fermi level corresponding to the HOMO and LUMO levels. However, CSW-479 also possesses states associated with the bipyridine side group, which are weakly coupled to the backbone and produce an additional Fano resonance in the electron transmission coefficients. ${ }^{17,18}$ The position of the
Fano resonance can be tuned via a variety of mechanisms, including ring rotation of the side group ${ }^{18}$ and gating by nearby polar molecules such as water, ${ }^{19}$ whereas BreitWigner resonances are relatively insensitive to such effects. In what follows, we demonstrate that changing the conformation of the side group in CSW-479 moves the Fano resonance close to the Fermi energy and creates huge changes in the magnitudes of the thermopower and can even change its sign, whereas only small changes occur when the conformation the BPDT molecule is changed.

The thermal and electrical properties of a molecular device can be understood by studying the thermoelectric coefficients $G, L, M$, and $K$, which relate the charge current $I$ and heat current $\dot{Q}$ to the electrical bias $\Delta V$ and the temperature difference $\Delta T$ across the system. The equations $I=G \Delta V$ $+L \Delta T$ and $\dot{Q}=M \Delta V+K \Delta T$ are commonly rewritten in terms of measurable thermoelectric coefficients: the electrical conductance $G$, thermopower $S$, Peltier coefficient $\Pi$, and thermal conductance $\kappa$, defined by

$$
\left(\begin{array}{c}
\Delta V \\
\dot{Q}
\end{array}\right)=\left(\begin{array}{cc}
R & S \\
\Pi & \kappa
\end{array}\right)\left(\begin{array}{c}
I \\
\Delta T
\end{array}\right) .
$$

Thermoelectric properties can therefore be utilized in either charge-driven cooling devices or heat-driven current generators. In conventional devices the maximum efficiency of either heat transfer or current generation is proportional to the dimensionless thermoelectric figure of merit $Z T=\frac{S^{2} G T}{\kappa}$ defined in terms of the measurable thermoelectric coefficients, where high efficiency corresponds to values $Z T \gg 1$. Some of the highest measured and predicted values of $S$ and $Z T$ are found in materials that have sharp DOSs (Refs. 20 and 21) such as bismuth nanowires ${ }^{22}$ or heavily doped semiconductors. $^{23}$ It is therefore expected that similar high values could be obtained in molecular devices since molecules also have sharp DOS close to their molecular levels.

The thermoelectric coefficients $S, G$, and $\kappa$ can be computed by extending the Landauer-Büttiker formalism to include both charge and heat currents. In the linear temperature and bias regime the three required thermoelectric coefficients $^{24}$ can be expressed in terms of the moments $L_{n}$ of the transmission coefficient via the following equations, ${ }^{25}$

$$
S=-\frac{1}{e T} \frac{L_{1}}{L_{0}},
$$




$$
\begin{gathered}
G=\frac{2 e^{2}}{h} L_{0}, \\
\kappa=\frac{2}{h} \frac{1}{T}\left(L_{2}-\frac{L_{1}^{2}}{L_{0}}\right), \\
Z T=\frac{1}{\frac{L_{0} L_{2}}{L_{1}^{2}}-1}, \\
L_{n}=\int_{-\infty}^{\infty}\left(E-E_{f}\right)^{n} \mathcal{T}(E) \frac{\partial f(E)}{\partial E} d E .
\end{gathered}
$$

If $T(E)$ is a slowly varying function of $E$, then at low temperatures, these expressions can be simplified by expanding $T(E)$ about $E=E_{F}$. In the present case, where $T(E)$ varies rapidly when a Fano resonance is present, we calculate the full integrals rather than taking the low-temperature limit.

To calculate thermoelectric properties from first principles, we use the density-functional theory code SIESTA (Ref. 26) which employs norm-conserving pseudopotentials to remove the core electrons ${ }^{27}$ and a local atomic-orbital basis set. In particular we use a double-zeta polarized basis set for all atoms and the local-density approximation for the exchange and correlation functional as parametrized by Ceperley and Alder. ${ }^{28}$ The Hamiltonian and overlap matrices are calculated on a real-space grid defined by a plane-wave cutoff of 200 Ry. Each molecule is relaxed into the optimum geometry until the forces on the atoms are smaller than $0.02 \mathrm{eV} / \AA$ and then sections of the molecules are rotated to the desired angles. The contact atoms are attached to the hollow site of a gold (111) surface consisting of 6 layers with 25 atoms/layer. The transmission coefficients are found by using the nonequilibrium Green's-function formalism. ${ }^{29}$

The large values of $S$ and $Z T$ predicted below rely only on the ability to move the Fano resonance close to the Fermi energy and are independent of the physical mechanism used to produce this shift. Furthermore, even though DFT may not predict the exact position of the Breit-Wigner and Fano resonances, the fact that the side groups are also aromatic groups and have energy levels close to the energy levels of the backbone, ensures the presence of Fano resonances near the Fermi level. Therefore an adjustment to the environment of the side group will generate the required shift. In what follows we examine the effect of rotating either the phenyl rings in BPDT or the side group in CSW-479, which could be induced by tilting the molecule using an STM tip ${ }^{3}$ or via steric hindrance. ${ }^{30-33}$

The sections of the molecules to rotate are chosen so that in BPDT the coupling along the backbone and in CSW-479 the coupling to the side group (see Fig. 1) is changed. For BPDT the molecule is first relaxed and then the torsion angle between the phenyl rings is artificially increased from $0^{\circ}$ to $90^{\circ}$, where $0^{\circ}$ corresponds to the case where the rings are planar. For CSW-479 the side group is rotated from $0^{\circ}$ to $180^{\circ}$ about the $\mathrm{C}-\mathrm{C}$ bond that couples it to the backbone,

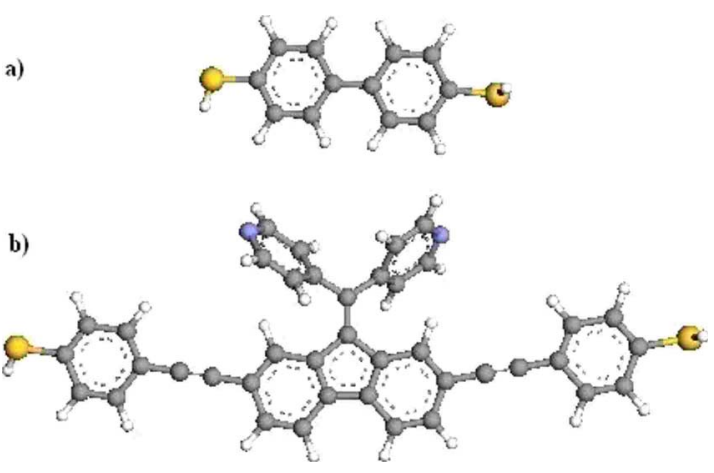

FIG. 1. (Color online) Compositions of the studied molecules (a) BPDT and (b) CSW-479.

where $0^{\circ}$ corresponds to when the bipyridine side group is approximately in line with the molecular backbone.

Figure 2 shows the transmission coefficients for (a) BPDT and (b) CSW-479 as a function of energy for different twist angles when the molecules are connected to the hollow site on the gold surfaces. As the phenyl rings are rotated from $0^{\circ}$ to $60^{\circ}$ in BPDT, the conjugation of the molecular backbone is reduced, which moves the LUMO level up in energy and reduces the transmission at the Fermi energy, following a known $\cos ^{2}(\theta)$ law. ${ }^{31-33}$ However the overall shape of the transmission remains almost unchanged. In contrast the CSW-479 molecule possesses a Fano resonance near the Fermi energy and as the side group is rotated, the Fano resonance moves to a lower energy, leading to a significant change in the slope of $T(E)$ near $E=E_{F}$.

For each twist angle, Fig. 3 shows the thermopower for (a) CSW-479 and (b) BPDT, obtained by using the transmission coefficients shown in Fig. 2, to evaluate Eqs. (2) and (6). The thermopower in the BPDT system increases smoothly with rotation angle at high temperatures and remains positive. This is expected since around the Fermi energy the transmission coefficient decays slowly for all angles and therefore the asymmetric term $L_{1}$ in Eq. (2) is always negative. The thermopower for the optimized geometry with $\theta \simeq 35^{\circ}$ is calculated to be $6.49 \mu \mathrm{V} \mathrm{K}^{-1}$, which is close to the value measured in Ref. 15. The discrepancy could be attributed to differences in the contact geometry. In fact, we know from previous calculations that by altering the contact configuration or by varying the distance between the leads and the molecule, ${ }^{34,35}$ the transmission coefficients can change dramatically, which modifies the slope near the Fermi
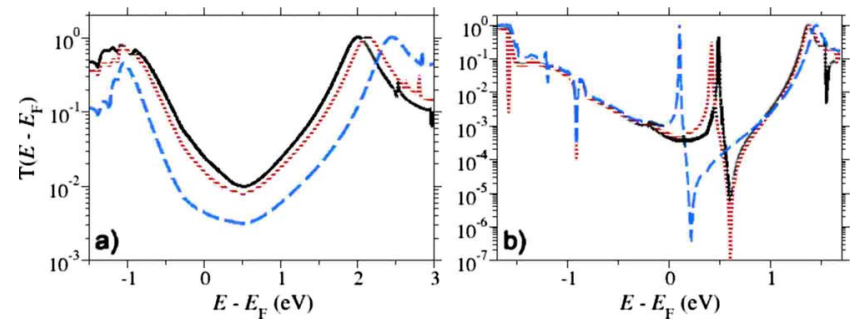

FIG. 2. (Color online) Transmission coefficient for the (a) BPDT and (b) CSW-479 molecules for twist angles of $0^{\circ}$ (continuous line), $30^{\circ}$ (dotted line), and $60^{\circ}$ (dashed line). 

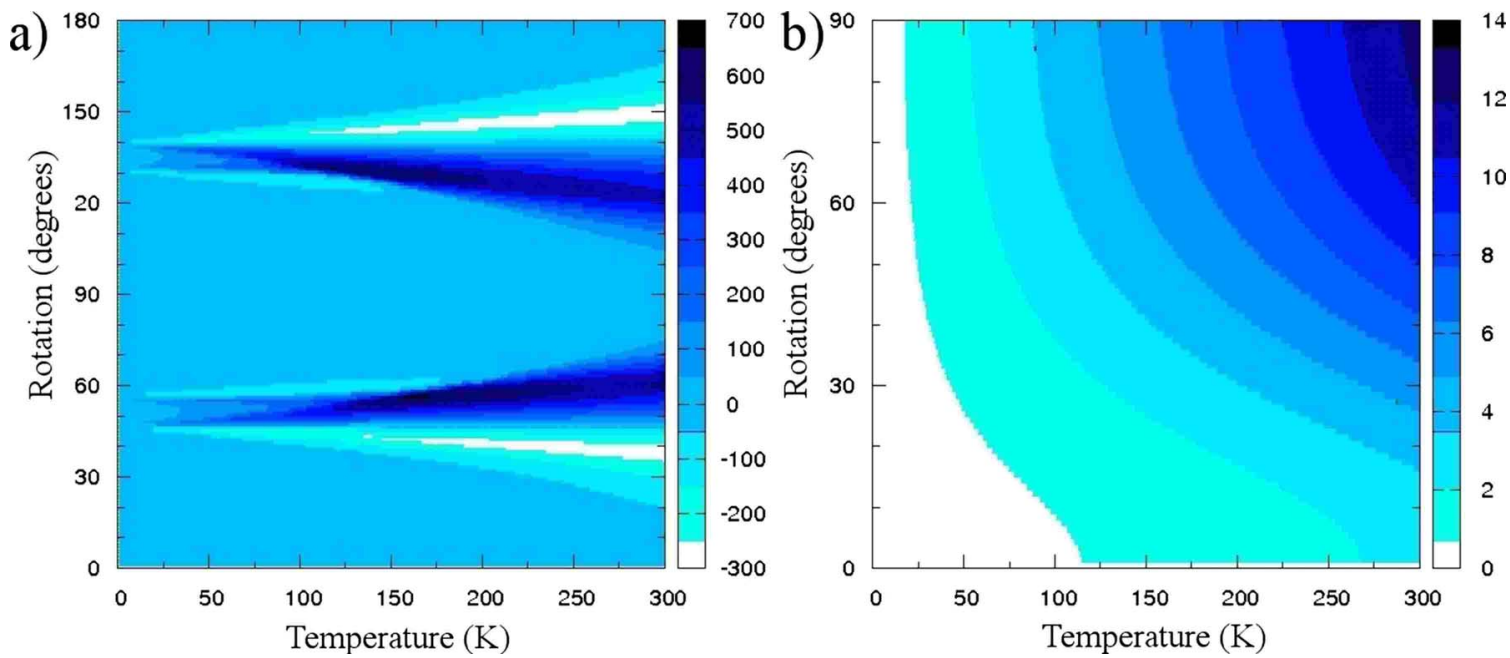

FIG. 3. (Color online) Contour plot of the thermopower $S$ for (a) CSW-479 and (b) BPDT as a function of temperature and twist angle. Note that the color scales used in the two figures differ by orders of magnitude.

level. Predicted thermopowers can also differ from experimental values even when the contact configuration is correct, due to approximations built into DFT.

In contrast to BPDT, the CSW-479 molecule produces large magnitudes for the thermopower when the Fano resonance is within $k_{B} T$ of the Fermi energy. At low temperatures $(T<50 \mathrm{~K})$, the largest value of $S$ is found to be approximately $200 \mu \mathrm{V} \mathrm{K}^{-1}$ and at higher temperatures of approximately $670 \mu \mathrm{V} \mathrm{K}^{-1}$. Furthermore the thermopower becomes negative as the angle increases and the Fano resonance approaches the Fermi energy from above. This suggests that thermoelectric properties can be tuned by modifying side groups and their coupling to the backbone. In this case it is also possible to obtain a negative thermopower at high temperatures even though the Fermi energy is still inside the HOMO-LUMO gap. The sign change in the thermopower arises from a combination of the asymmetric term $L_{1}$ and the intrinsic asymmetric shape of the Fano resonance. Therefore, the idea that the hole or electronlike character of conduction is determined by the sign of the thermopower is ambiguous for systems with Fano resonances close to the Fermi energy.

As a demonstration of the generic nature of these predictions, we repeated the above calculations for molecules connected to a gold atom on top of a gold electrode. The results were qualitatively similar, even though the details changed, such as the position of the Fermi energy, which shifted slightly closer to the HOMO level due to the reduction in the charge transfer in the top configuration.

Figure 4 shows the calculated dimensionless figures of merit for (a) CSW-479 and (b) BPDT. Due to the low thermopower and conductance near the Fermi level the figure of merit for BPDT is low for all rotation angles, and therefore this molecule has a low efficiency of thermoelectric heat transfer. This is not the case for CSW-479, where even at low temperatures a figure of merit $Z T \gg 1$ can be achieved for rotation angles where the Fano resonance is close to the Fermi energy. This demonstrates that the temperature difference can be mechanically tuned by adjusting the coupling
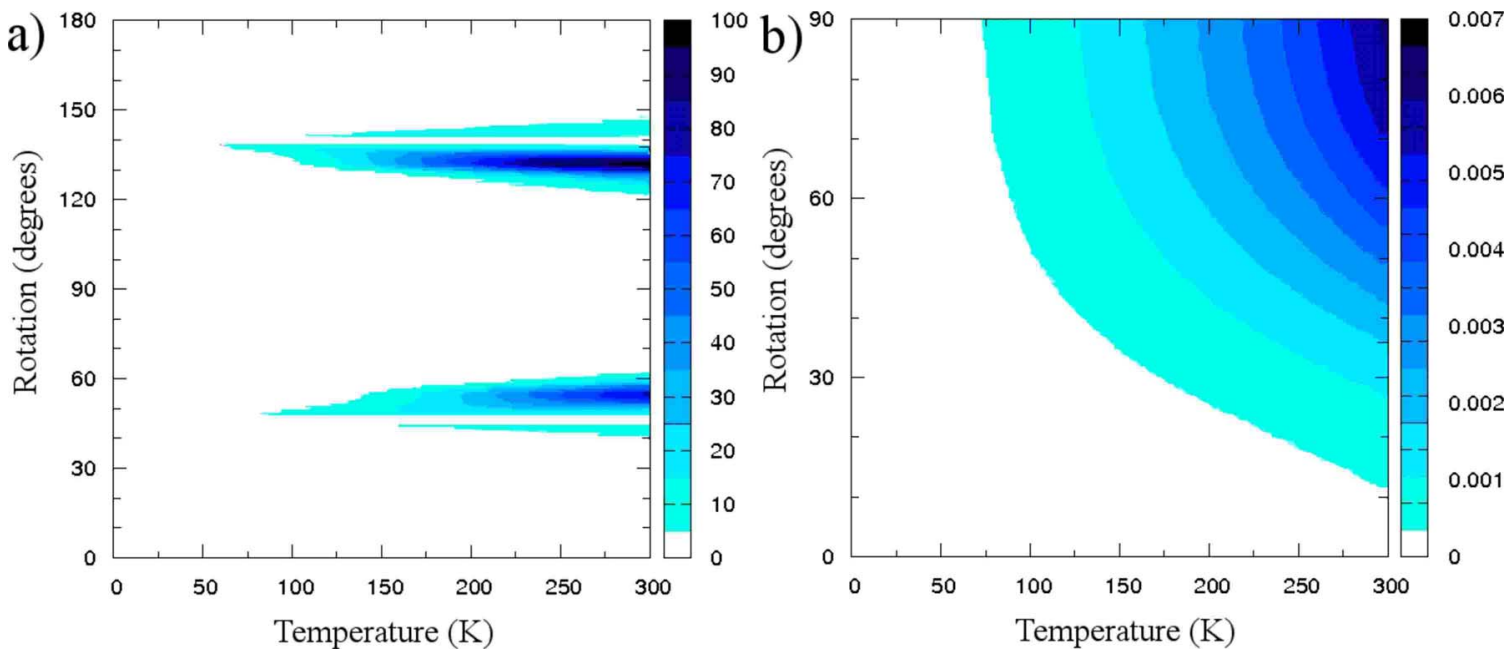

FIG. 4. (Color online) Contour plot of the figure of merit ZT for (a) CSW-479 and (b) BPDT as a function of temperature and twist angle. 
between the localized states and the backbone. Such large figures of merit have been predicted for semiconducting carbon nanotubes. ${ }^{36}$ However to date this is the largest predicted figure of merit value for an ungated single molecular device.

We have shown there that is a huge difference in the thermoelectric properties of molecules with and without side groups. In contrast with electrical properties, the thermoelectric properties of a molecular device depend on the shape of the transmission profile about the Fermi energy rather than its absolute magnitude. By moving a Fano resonance through the Fermi energy, the shape of the transmission can be dramatically altered to produce both huge thermopower and fig- ures of merit, corresponding to a high-efficiency thermoelectric cooling device. In practice, at high temperatures, this value could be limited by the contribution to the thermal conductance from phonons, ${ }^{37}$ which acts in parallel to the electronic contribution to the thermal conductance and is not included here. Electron transport through quasi-onedimensional wires is also sensitive to disorder, ${ }^{38,39}$ which could arise under ambient conditions, due to the presence of polar water molecules. ${ }^{19}$

We would like to acknowledge funding from the EPSRCGB, the NWDA, the DTI, and the MCRTN "FUNMOLS." *c.lambert@lancaster.ac.uk

${ }^{1}$ H. B. Weber, J. Reichert, F. Weigend, R. Ochs, D. Beckmann, M. Mayor, R. Ahlrichs, and H. v. Löhneysen, Chem. Phys. 281, 113 (2002).

${ }^{2}$ B. Xu and N. J. Tao, Science 301, 1221 (2003).

${ }^{3}$ Wolfgang Haiss, Changsheng Wang, Iain Grace, Andrei S. Batsanov, David J. Schiffrin, Simon J. Higgins, Martin R. Bryce, Colin J. Lambert, and Richard J. Nichols, Nature Mater. 5, 995 (2006).

${ }^{4}$ B.-Y. Choi, S.-J. Kahng, S. Kim, H. Kim, H. W. Kim, Y. J. Song, J. Ihm, and Y. Kuk, Phys. Rev. Lett. 96, 156106 (2006).

${ }^{5}$ E. Lörtscher, J. W. Ciszek, J. Tour, and H. Reil, Small 2, 973 (2006).

${ }^{6}$ G. J. Ashwell, A. Mohib, and J. R. Miller, J. Mater. Chem. 15, 1160 (2005)

${ }^{7}$ G. J. Ashwell, W. D. Tyrrell, B. Urasinska, C. Wang, and M. R. Bryce, Chem. Commun. (Cambridge) 2006, 1640 (2006).

${ }^{8}$ J. He, B. Chen, A. K. Flatt, J. J. Stephenson, C. D. Doyle, and J. M. Tour, Nature Mater. 5, 63 (2006).

${ }^{9}$ D. P. Long, J. L. Lazorcik, B. A. Mantooth, M. H. Moore, M. A. Ratner, A. Troisi, Y. Yao, J. W. Ciszek, J. M. Tour, and R. Shashidhar, Nature Mater. 5, 901 (2006).

${ }^{10}$ J. Koch, F. von Oppen, Y. Oreg, and E. Sela, Phys. Rev. B 70, 195107 (2004).

${ }^{11}$ M. Paulsson and S. Datta, Phys. Rev. B 67, 241403(R) (2003).

${ }^{12}$ F. Pauly, J. K. Viljas, and J. C. Cuevas, Phys. Rev. B 78, 035315 (2008).

${ }^{13}$ B. Wang, Y. Xing, L. Wan, Y. Wei, and J. Wang, Phys. Rev. B 71, 233406 (2005).

${ }^{14}$ X. Zheng, W. Zheng, Y. Wei, Z. Zeng, and J. Wang, J. Chem. Phys. 121, 8537 (2004).

${ }^{15}$ P. Reddy, S. Jang, R. A. Segalman, and A. Majumdar, Science 315, 1568 (2007).

${ }^{16}$ C. Wang, A. S. Batsanov, and M. R. Bryce, J. Org. Chem. 71, 108 (2006).

${ }^{17}$ D. M. Cardamone, C. A. Stafford, and S. Mazumdar, Nano Lett. 6, 2422 (2006).

${ }^{18}$ T. A. Papadopoulos, I. M. Grace, and C. J. Lambert, Phys. Rev. B 74, 193306 (2006).

${ }^{19}$ E. Leary et al. (unpublished).
${ }^{20}$ G. D. Mahan and J. O. Sofo, Proc. Natl. Acad. Sci. U.S.A. 93, 7436 (1996).

${ }^{21}$ L. D. Hicks and M. S. Dresselhaus, Phys. Rev. B 47, 16631 (1993).

${ }^{22}$ S. B. Cronin, Y.-M. Lin, M. R. Black, O. Rabin, and M. S. Dresselhaus, XXI International Conference on Thermoelectrics, Proceedings ICT '02, 2002 (unpublished), pp. 243-248.

${ }^{23}$ Y.-M. Lin, X. Sun, and M. S. Dresselhaus, Phys. Rev. B 62, 4610 (2000).

${ }^{24}$ Here, $\kappa$ includes only the electric contribution to the thermal conductance, with the phonon term neglected as in Ref. 13.

${ }^{25}$ N. R. Claughton and C. J. Lambert, Phys. Rev. B 53, 6605 (1996).

${ }^{26}$ J. M. Soler, E. Artacho, J. D. Gale, A. Garcia, J. Junquera, P. Ordejon, and D. Sanchez-Portal, J. Phys.: Condens. Matter 14, 2745 (2002).

${ }^{27}$ N. Troullier and J. L. Martins, Phys. Rev. B 43, 1993 (1991).

${ }^{28}$ D. M. Ceperley and B. J. Alder, Phys. Rev. Lett. 45, 566 (1980).

${ }^{29}$ A. R. Rocha, V. M. García-Suárez, S. Bailey, C. Lambert, J. Ferrer, and S. Sanvito, Phys. Rev. B 73, 085414 (2006).

${ }^{30}$ L. Venkataraman, J. E. Klare, C. Nuckolls, M. S. Hybersten, and M. L. Steigerwald, Nature (London) 442, 904 (2006).

${ }^{31}$ C. M. Finch, S. Sirichantaropass, S. W. Bailey, I. M. Grace, V. M. García-Suárez, and C. J. Lambert, J. Phys.: Condens. Matter 20, 022203 (2008).

${ }^{32}$ L. Venkataraman, J. E. Klare, C. Nuckolls, M. S. Hybertsen, and M. L. Steigerwald, Nature (London) 442, 904 (2006).

${ }^{33}$ S. Woitellier, J. P. Launay, and C. Joachim, Chem. Phys. 131, 481 (1989)

${ }^{34}$ V. M. García-Suárez, T. Kostyrko, S. Bailey, C. Lambert, and B. R. Bułka, Phys. Status Solidi B 244, 2443 (2007).

${ }^{35}$ R. C. Hoft, M. J. Ford, V. M. García-Suárez, C. J. Lambert, and M. B. Cortie, J. Phys.: Condens. Matter 20, 025207 (2008).

${ }^{36}$ K. Esfarjani, M. Zebarjadi, and Y. Kawazoe, Phys. Rev. B 73, 085406 (2006).

${ }^{37}$ P. Murphy, S. Mukerjee, and J. Moore, Phys. Rev. B 78, 161406 (2008).

${ }^{38}$ C. J. Lambert, J. Phys. C 17, 2401 (1984).

${ }^{39}$ C. J. Lambert, Phys. Rev. B 29, 1091 (1984). 